\title{
PROTOCOLO DE INDUÇÃO DE CETOSE SUBCLÍNICA E SEU EFEITO SOBRE PARÂMETROS BIOQUÍMICOS EM OVELHAS GESTANTES
}

\footnotetext{
${ }^{1}$ Doutoranda em Medicina Veterinária, UFPEL; ${ }^{2}$ Graduanda em Medicina Veterinária, UFPEL; ${ }^{3}$ Pós-Doutor em Medicina Veterinária, UFPEL; ${ }^{4}$ Professor Adjunto no Departamento de Clínicas Veterinárias, UFPEL; ${ }^{5}$ Professor Associado do Centro de Ciências Químicas, Farmacêuticas e de Alimentos, UFPEL; ${ }^{6}$ Professor da Universidade Anhanguera-Uniderp-Campo Grande, MS; ${ }^{7}$ Professora Adjunta no Departamento de Clínicas Veterinárias, UFPEL; ${ }^{8}$ Professor Associado no Departamento de Clínicas Veterinárias, UFPEL.
}

\section{RESUMO}

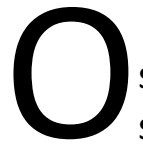
objetivo deste estudo foi avaliar a eficiência de um protocolo de indução de cetose subclínica em ovelhas do grupo genético pantaneiro no terço final da gestação e o seu efeito sobre parâmetros metabólicos. Foram utilizadas 13 ovelhas gestantes, com idade de dois a quatro anos, com aproximadamente 97 dias de gestação, peso corporal de $40 \pm 5 \mathrm{~kg}$ e escore de condição corporal entre dois e três no início do experimento. Durante quatro dias, dos 132 aos 136 dias de gestação, os animais foram submetidos a uma restrição alimentar da ordem de $70 \%$ das necessidades nutricionais da categoria. Amostras de sangue foram coletadas a partir dos 97 dias de gestação até 56 dias pós-parto. Foram avaliados os níveis séricos de glicose, colesterol total, aspartato aminotransferase (AST), gama glutamil transferase (GGT), albumina, ureia, fósforo, ácidos graxos não esterificados (AGNE) e beta-hidroxibutirato (BHB). Durante o período de indução, alterações metabólicas significativas foram observadas na maioria dos metabólitos analisados. Os níveis séricos de glicose $(p<0,0001)$ e fósforo $(p<0,001)$ diminuíram, enquanto as concentrações de AGNE $(p<0,0001)$, colesterol $(p<0,001), G G T(p<0,001)$ e ureia $(p<0,001)$ aumentaram. Não foram observados sinais clínicos que indicassem o desenvolvimento do quadro clínico da doença. Concluímos que o protocolo avaliado neste estudo foi eficaz na indução da cetose subclínica durante quatro dias e os parâmetros analisados podem ser úteis para predizer a forma clínica da doença, na ovelha da raça pantaneira.

Palavras-chave: Grupo genético pantaneiro. Restrição alimentar. Toxemia da prenhez. 


\section{INTRODUÇÃO}

Cetose ou toxemia da prenhez é uma das principais causas de mortalidade de ovelhas durante o período final da gestação (terço final), é nesse período que há aumento das exigências nutricionais para atender as suas necessidades e as do(s) feto(s) em formação (BROZOS et al., 2011). A cetose é um desequilíbrio metabólico que acomete principalmente ovelhas pluríparas e com gestações gemelares (MOALLEM et al., 2012). Esta doença é caracterizada por mobilização lipídica durante um período em que há alta necessidade de glicose, a qual não pode ser suprida pela alimentação, pois a glicose está sendo direcionada para a glândula mamária, podendo resultar em hipoglicemia, aumento dos níveis séricos de ácidos graxos não esterificados (AGNE) e corpos cetônicos: como acetona, acetoacetato e beta-hidroxibutirato (BHB) (HARMEYER; SCHLUMBOHM, 2008).

A cetose pode ter uma manifestação subclínica, com uma incidência superior a $20 \%$ em rebanhos criados em sistemas intensivos (LACETERA et al., 2001). Aumentando as taxas de aborto, além de predispor ao desenvolvimento de outras doenças, como hipocalcemia e acidose metabólica (MOALLEM et al., 2012).

A doença pode ser classificada conforme o seu grau de severidade, de acordo com o perfil bioquímico do BHB e sinais clínicos. Taghipour et al. (2010) definiram que valores entre 0,8 e 1,6 mmol/L de BHB são indicativos de cetose subclínica e presença de balanço energético negativo (BEN), enquanto que níveis superiores a $1,6 \mathrm{mmol} / \mathrm{L}$ podem ser considerados indicativos de cetose clínica. A partir desse nível já é possível a observação de sinais clínicos como: letargia, hipofagia, anorexia e hiperexcitabilidade (CAMPOS et al., 2010).

Para melhor compreender a doença, experimentos baseados em completa restrição alimentar vêm sendo realizados para induzir a cetose na sua forma clínica (EMMANUEL; NAHAPETIAN, 1975; LEKATZ et al., 2011; RANAWEERA et al., 1979; WEST, 1996).

Um protocolo de indução de cetose subclínica que possibilite um estudo mais detalhado da enfermidade e a determinação dos parâmetros metabólicos que se alteram precocemente, permitiria a monitoração do status nutricional das ovelhas no pré-parto, evitando-se a manifestação da enfermidade na sua forma clínica e subclínica. Entretanto, os protocolos de indução existentes apresentam algumas limitações quanto a essa aplicabilidade, uma vez 
que os animais desenvolvem o quadro clínico da doença muito facilmente, pois a margem entre a forma clínica e subclínica é muito tênue (KANEKO et al., 2008; MEIRA JR. et al., 2009).

Diante disso, o objetivo deste estudo foi avaliar a eficiência de um protocolo de indução de cetose subclínica em ovelhas do grupo genético pantaneiro, no terço final de gestação, e o seu efeito sobre parâmetros metabólicos nutricionais.

\section{MATERIAL E MÉTODOS}

Todos os procedimentos experimentais foram aprovados pelo Comitê de Ética de Experimentação Animal da Universidade Federal de Pelotas (Código 7933).

\section{Animais}

Este estudo foi realizado no Centro Tecnológico de Ovinocultura da Universidade

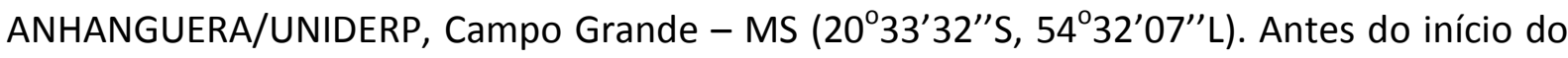
experimento, 40 ovelhas do grupo genético pantaneiro foram submetidas a um protocolo de sincronização de cio, com o uso de um dispositivo intravaginal (Eazi-Breed CIDR ${ }^{\circledR}$, Pfizer Saúde Animal, Brasil) por 11 dias. No momento da retirada do dispositivo foi aplicado, por via intramuscular, 500 UI de gonadotrofina coriônica equina (Folligon ${ }^{\circledR} 5000$ UI, Intervet Schering Plough, Brasil). A expressão do cio destas fêmeas foi monitorada nos cinco dias seguintes, através de rufiões, e após a identificação foram submetidas à monta controlada. O diagnóstico de gestação foi realizado 45 dias após a monta, através de ultrassonografia transcutânea (ANSER VET 450, Pie Medical).

Após a comprovação da gestação, foram selecionadas 13 ovelhas gestantes com idade entre dois e quatro anos, peso corporal médio de $40 \pm 5 \mathrm{~kg}$ e escore de condição corporal entre dois e três no início do experimento (RUSSEL et al., 1969).

Todos os animais foram submetidos ao mesmo protocolo nutricional, permanecendo em pastagem do gênero Brachiaria brizantha cv. Marandu (800 kg de matéria seca/ha, 8,4\% de proteína bruta (PB), 65\% de fibra detergente neutro (FDN) e 40\% de fibra detergente ácido (FDA)) até os 103 dias de gestação, sendo acrescida à dieta, aos 104 dias de gestação, 1\% do peso vivo (PV) de concentrado (Nutri ovinos confinamento - Agrolima Nutrição Animal, 
Campo Grande, MS, Brasil) (Tabela 1). Aos 111 dias de gestação o nível de concentrado foi aumentado para 2\% do PV. Dos 132 dias aos 136 dias de gestação os animais foram submetidos à restrição alimentar (período de indução de cetose subclínica), consumindo apenas silagem de milheto ( $16 \%$ de PB, $30 \%$ de FDN e $16,4 \%$ de FDA) correspondendo a $30 \%$ da necessidade de ingestão de matéria seca (IMS) para a categoria, de acordo com o NRC (2001). Após o período de restrição foi restabelecida a dieta à base de concentrado em quantidade equivalente a $2 \%$ do PV, até o momento do parto. No pós-parto os animais retornaram a pastagem consumindo apenas Brachiaria brizantha cv. Marandu.

Tabela 1 - Composição bromatológica do concentrado comercial fornecido para as ovelhas no período experimental, no ano de 2011 - Brasil.

$\begin{array}{cc}\text { Componente } & \text { Quantidade por kg } \\ \text { Matéria Seca } & 120 \mathrm{~g} \\ \text { Proteína Bruta } & 125 \mathrm{~g} \\ \text { Fibra } & 14,40 \mathrm{~g} \\ \text { Mineral } & 150 \mathrm{~g} \\ \text { Cálcio } & 43 \mathrm{~g} \\ \text { Fósforo } & 8,300 \mathrm{mg} \\ \text { Cobalto } & 3,000 \mathrm{mg} \\ \text { Magnésio } & 0,40 \mathrm{mg} \\ \text { Selênio } & 50 \mathrm{mg}\end{array}$

Fonte: Nutri ovinos confinamento - Agrolima Nutrição Animal, Campo Grande, MS, Brasil.

\section{Coletas de sangue e análises metabólicas}

O experimento foi subdividido em quatro períodos: pré-indução (dos 97 até 131 dias de gestação); indução (dos 132 até 136 dias de gestação); pós-indução (dos 137 até 142 dias de gestação) e pós-parto (dia 0 até 56 dias pós-parto). Todas as amostras de sangue foram coletadas através de venopunção, utilizando acoplador e agulha $25 \times 7 \mathrm{~mm}$ (Agulha BD Vacutainer $^{\circledast}$ Eclipse $^{\mathrm{TM}}$ - Brasil). Durante o período de pré-indução, as amostras foram coletadas com intervalos de sete dias, a partir de 97 dias de gestação. No período de indução 
de cetose subclínica, as amostras foram coletadas duas vezes ao dia (uma coleta no turno matutino e outra no vespertino). No período pós-indução, as amostras foram coletadas de três em três dias até o dia do parto (os partos ocorreram com aproximadamente $142 \pm 3$ dias de gestação), após o parto as amostras foram coletadas com intervalos de sete dias até 56 dias pós-parto.

As amostras de sangue foram coletadas em dois tubos, um com ácido etilenodiaminotetracético (EDTA) e fluoreto de potássio ( $4 \mathrm{~mL}$ Vacuplast ${ }^{\circledR}$ - Zhejiang, China) para obtenção de plasma para posterior análise de glicose e outro sem anticoagulante (10 $\mathrm{mL}$ Vacuplast $^{\circledR}$ - Shandong, China) para obtenção do soro, sendo centrifugados a $1.000 \times \mathrm{g}$ durante 15 minutos, e criopreservadas a $-80{ }^{\circ} \mathrm{C}$ para posterior análise dos metabólitos: colesterol total, aspartatoaminotransferase (AST), gama glutamil transferase (GGT), albumina, ureia, fósforo, BHB e ácidos graxos não esterificados (AGNE), estas foram realizadas com auxílio de kits de diagnóstico espectofotométrico (Labtest Diagnóstica S. A., Brasil), utilizando fotocolorimetria em espectrofotômetro de luz visível (FEMTO $435^{\circledR}$, Brasil). As análises de AGNE e BHB foram realizadas através do método descrito por Ballou et al. (2009), utilizando um kit comercial (Wako, USA, Richmond, USA e Ranadox, Oceanside, CA, respectivamente). Os coeficientes de variação foram inferiores a 10\% para todos os ensaios.

Durante o experimento os animais foram monitorados diariamente e determinadas as frequências cardíaca, respiratória e ruminal, além da temperatura retal e coloração das mucosas, segundo descrito por Leydson (2008).

\section{Análise estatística}

Os resultados foram analisados pelo programa SAS ${ }^{\circledR}$ (2009), através de ONE WAY ANOVA, com comparação entre médias de acordo com o Teste de Tukey HSD $(p<0,05)$ para os metabólitos (níveis séricos de glicose, colesterol, ureia, albumina, fósforo, AGNE, AST, GGT e BHB) entre os períodos de avaliação (pré-indução, indução, pós-indução e pós-parto).

\section{RESULTADOS E DISCUSSÃO}

No período de pré-indução, todos os parâmetros bioquímicos analisados estavam de acordo com os valores de referência fisiológicos para a espécie ovina: glicose $57,18 \pm 1,79 \mathrm{mg} / \mathrm{dL}$ (50 
a $80 \mathrm{mg} / \mathrm{dL}$ ) (GONZÁLEZ et al., 2006; CALDEIRA, 2005), BHB 0,28 $\pm 0,26 \mathrm{mmol} / \mathrm{L}(0,1 \mathrm{a} 0,7$

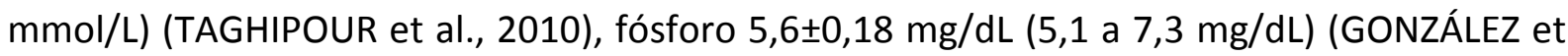
al., 2006), colesterol 76,56 $\pm 2,79 \mathrm{mg} / \mathrm{dL}$ (52 a $76 \mathrm{mg} / \mathrm{dL}$ ) (GONZÁLEZ et al., 2006), albumina

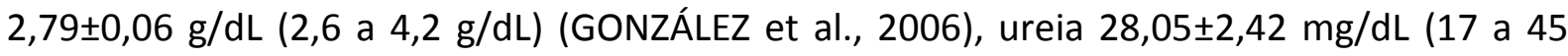

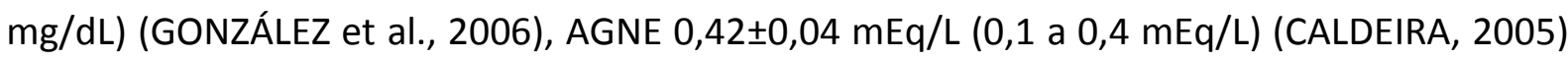
e para as enzimas; AST $84,7 \pm 3,7 \mathrm{U} / \mathrm{L}$ (53,83 a 107,65 U/L) (EL-SHERIF; ASSAD, 2001) e GGT $55,13 \pm 2,3$ (20 a 56 U/L) (GONZÁLEZ et al., 2006) (Figura 1).

No período de indução de cetose subclínica houve aumento nos níveis de $\operatorname{AGNE}(1,13 \pm 0,04$ $\mathrm{mEq} / \mathrm{L}, \mathrm{p}<0,0001)$, ureia $(56,94 \pm 2,54 \mathrm{mg} / \mathrm{dL}, \mathrm{p}<0,0001)$, GGT $(142,74 \pm 9,03 \mathrm{U} / \mathrm{L}, \mathrm{p}<0,0001)$ e colesterol $(86,13 \pm 2,33 \mathrm{mg} / \mathrm{dL}, \mathrm{p}<0,001)$; e queda nos níveis de glicose $(33,01 \pm 1,27 \mathrm{mg} / \mathrm{dL}$, $\mathrm{p}<0,0001)$ e fósforo $(4,13 \pm 0,19 \mathrm{mg} / \mathrm{dL}, \mathrm{p}<0,0001)$. Durante esse período apenas um animal demonstrou sinais clínicos de cetose, confirmado através do valor de BHB, superior a 1,6 $\mathrm{mmol} / \mathrm{L}$, sendo retirado do experimento. Já no período de pós-indução, houve aumento nos valores de glicose $(90,71 \pm 4,01 \mathrm{mg} / \mathrm{dL}, \mathrm{p}<0,0001)$, BHB $(1,52 \pm 0,48 \mathrm{mmol} / \mathrm{L}, p<0,0001)$ e GGT $(182,16 \pm 10,57 \mathrm{U} / \mathrm{L}, \mathrm{p}<0,0001)$. O BHB e a GGT permaneceram com concentrações elevadas até o pós-parto $(2,19 \pm 0,21 \mathrm{mmol} / \mathrm{L}$ e $179,63 \pm 9,54 \mathrm{U} / \mathrm{L}$, respectivamente).

No período do pós-parto observou-se aumento nos valores de AGNE $(0,81 \pm 0,03 \mathrm{mEq} / \mathrm{L}$, $p<0,0001)$ e ureia $(33,77 \pm 2,43, p<0,0001)$; e redução nos níveis de glicose $(70,78 \pm 3,62$ $\mathrm{mg} / \mathrm{dL}, \mathrm{p}<0,0001)$. Houve alterações nas concentrações de AST em todos os períodos, com concentrações mais altas no período de pré-indução $(84,71 \pm 3,70 U / L, p<0,0001)$ (Figura 1). Não foram observadas diferenças significativas nos níveis de albumina durante o

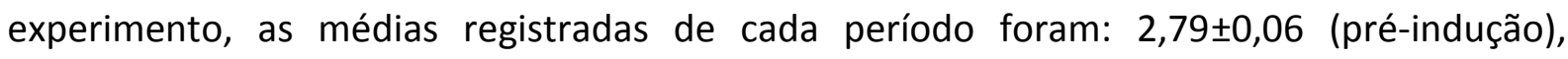

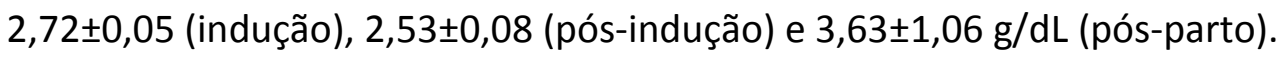


A
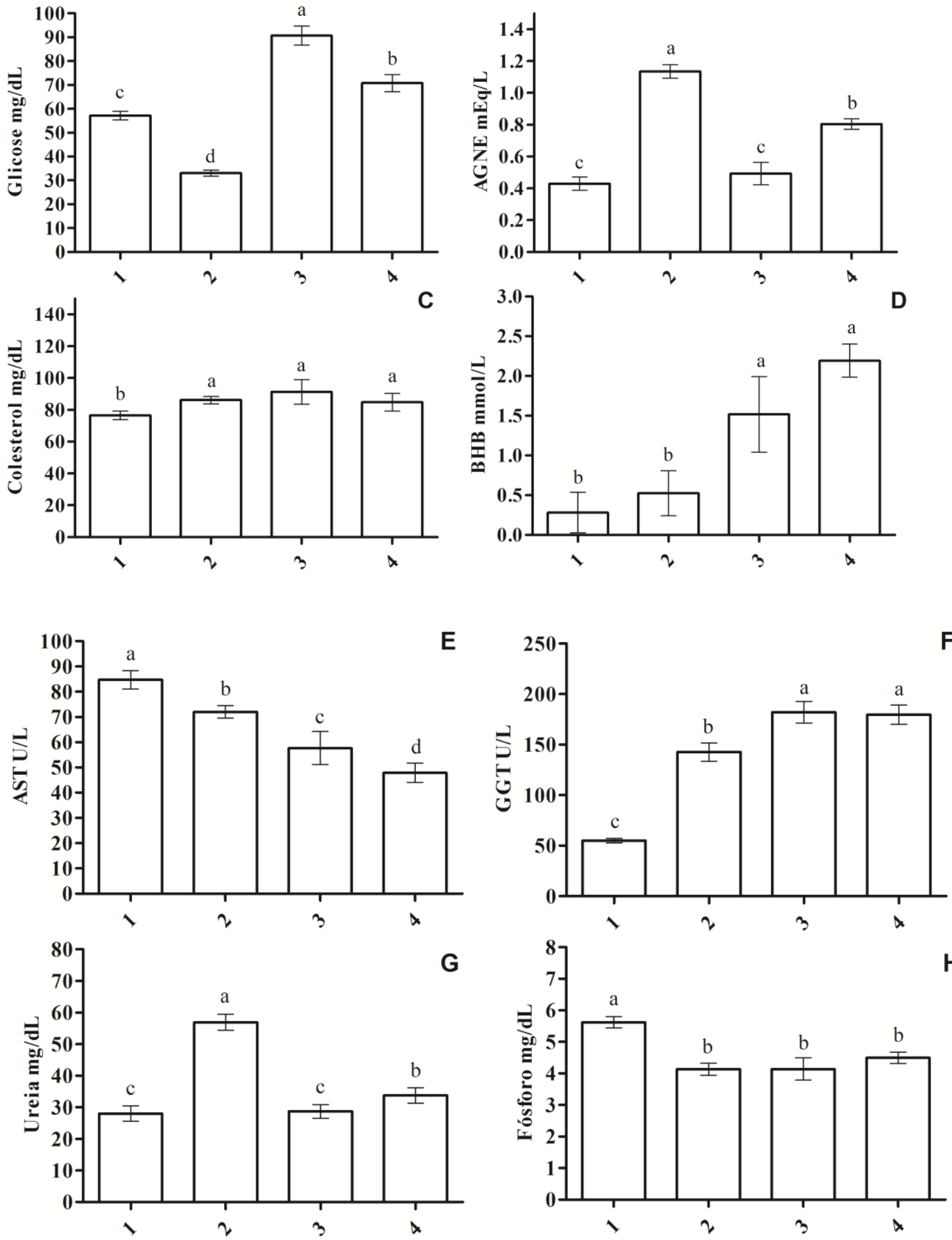

Figura 1 - Média e erro padrão da média de parâmetros metabólicos de ovelhas pantaneiras submetidas a um protocolo de indução de cetose. ${ }^{a, b, c, d}$ Letras minúsculas diferentes indicam diferença estatística $(p<0,05)$. Períodos: 1=Pré-indução, 2=Indução, 3=Pós-indução, 4=Pós-parto. 
Atualmente entre os parâmetros utilizados como indicadores nutricionais, destacam-se o AGNE, o BHB e a glicose como indicadores do status energético e a albumina e a ureia na avaliação do status protéico, de amostras tanto do soro como do plasma (CALDEIRA et al., 2007). Estudos realizados por Emmanuel e Nahapetian (1975) e West (1996), durante a utilização de um protocolo de indução de cetose, observaram hipoglicemia associada a cetonemia acentuada no período de indução o que justificaria a ocorrência da cetose na sua forma clínica. A hipoglicemia neste estudo foi causada pela restrição alimentar da ordem de $70 \%$ das necessidades nutricionais durante o momento de indução, o que acarretou a mobilização das reservas energéticas do organismo, elevando as concentrações de AGNE e BHB (HENZE et al., 1998). O grau de indução de cetose subclínica neste estudo não ultrapassou os limites de referência de AGNE (1,2 mEq/L) e BHB (1,6 mml/L) (TAGHIPOUR et al., 2010), para a manifestação do quadro clinico. Já no estudo de González et al. (2012), foi retirada toda a alimentação e induzida a cetose clínica em cabras, e observou-se que os níveis de BHB aumentaram após 48 horas. No presente estudo observou-se que o aumento dos níveis de BHB foi tardio, isso pode ser devido a não retirada total de alimento, ocorrendo o aumento da sua concentração somente após o período de indução, o que provavelmente colaborou para a não ocorrência da forma clínica da doença.

Quanto aos níveis séricos de colesterol total, este pode ser proveniente dos alimentos ou sintetizado a partir da acetil-CoA no fígado, mediante regulação da ingestão de colesterol exógeno. Quando ocorre a beta-oxidação das reservas lipídicas ocorre o aumento da acetilCoA (BOBE et al., 2004) e, concomitantemente, a elevação das concentrações de colesterol, o que explicaria o aumento do colesterol total no período de indução e a manutenção dos seus níveis elevados até o período pós-parto. Da mesma forma, as concentrações séricas de ureia aumentaram durante o período de indução, pois a restrição alimentar ativa a proteólise endógena para utilização de aminoácidos como fonte energética (REYNOLDS, 1992). Em animais submetidos a jejum, ocorre a diminuição da capacidade da microbiota ruminal em utilizar compostos nitrogenados para a síntese de proteínas, aumentando a quantidade de amônia absorvida no rúmen (CORRÊA et al., 2010). 
Cerca de $80 \%$ do fósforo apresenta-se como constituinte ósseo, enquanto que os outros $20 \%$ desempenham diferentes funções como composição dos ácidos nucléicos, essenciais no crescimento e na diferenciação celular, composição dos fosfolipídios das membranas celulares, atuando na permeabilidade e seletividade de membrana e como fosfato inorgânico, participando da manutenção do pH (VITTI; KEBREAB, 2010). Além disso, ele participa diretamente do metabolismo energético, atuando tanto no catabolismo como no anabolismo da glicose, pois é um dos principais constituintes da molécula de armazenamento de energia, a adenosina trifosfato (ATP) (GRÜNBERG et al., 2009). As concentrações de fósforo podem diminuir no início da lactação, devido a diminuição da ingestão de matéria seca (IMS) (GRÜNBERG et al., 2009), o que explicaria a diminuição da sua concentração, no presente estudo, no período de indução com a manutenção dos níveis diminuídos até o período de pós-parto.

Para avaliação da função hepática foram analisados os níveis séricos de AST, GGT e albumina, sendo que a enzima AST é utilizada como indicador de danos nos tecidos hepático, músculo esquelético e cardíaco. Porém neste estudo não observou-se aumento da atividade desta enzima, somente variações dentro dos limites fisiológicos, durante os períodos estudados. Já a enzima GGT possui maior atividade no córtex renal, medula renal, pâncreas, glândula mamária e fígado. Neste estudo, o aumento dos seus níveis durante o período de indução, foi proporcionado pela lipomobilização, comprovada pelo aumento dos níveis de AGNE e a consequente hiperatividade hepática (HENZE et al., 1998). A albumina é a proteína mais abundante no sangue, sendo sintetizada no fígado, suas concentrações plasmáticas são utilizadas como indicador da função hepática e do estado protéico (HOFFMAN et al., 2001). Neste estudo, não foi observada diferença significativa em nenhum momento nos níveis de albumina, provavelmente devido ao curto período de indução (132 aos 136 dias de gestação). Para a detecção de mudanças nos níveis de albumina sérica é necessário um período de restrição alimentar superior a 30 dias, devido à baixa velocidade de síntese e degradação (MEIRA et al., 2009).

A ovelha pantaneira ainda não é uma raça reconhecida, mas sim um grupo genético com características próprias adquiridas em função dos cruzamentos e da evolução natural 
(FERREIRA et al., 2012). Há poucos relatos sobre os parâmetros bioquímicos da raça, relacionados a produção e reprodução. Portanto a determinação do perfil bioquímico para identificação da cetose subclínica é fundamental como ferramenta diagnóstica, permitindo desta forma um diagnóstico precoce da enfermidade.

\section{CONCLUSÃO}

O protocolo de indução da cetose subclínica avaliado neste estudo foi eficaz no período de quatro dias. Os dados apresentados são importantes, colaborando na melhor compreensão da indução e manutenção da cetose subclínica, sem que os animais desenvolvam a forma clínica da doença. As informações aqui geradas podem servir de base para estudos futuros, possibilitando a quantificação das perdas causadas por esta desordem metabólica, particularmente no grupo genético pantaneiro.

\section{PROTOCOL OF INDUCTION OF SUBCLINICAL KETOSIS AND ITS EFFECT ON BIOCHEMICAL PARAMETERS IN PREGNANT EWES}

\section{ABSTRACT}

$\mathrm{T}$ he aim of this study was to evaluate the efficiency of a protocol of induction of subclinical ketosis in ewes from pantaneiro genetic group during the final three months of pregnancy and its effect on metabolic parameters. 13 pregnant ewes were used, they were aged between 2-4 years with approximately 97 days of pregnancy, body weight of $40 \pm 5 \mathrm{~kg}$ and body condition score between 2 and 3. For four days, from days 132 to 136 of pregnancy, the animals were subjected to feed restriction of $70 \%$ of the nutrient requirement. Blood samples were collected from day 97 of pregnancy until day 56 postpartum. Serum levels of glucose, total cholesterol, aspartate aminotransferase (AST), gamma glutamyl transferase (GGT), albumin, urea, phosphorus, non-esterified fatty acids (NEFA) and beta-hydroxybutyrate (BHB) were evaluated. During the induction period, significant metabolic changes were observed in most of the metabolites analyzed. Serum levels of glucose $(p<0.0001)$ and phosphorus $(p<0.001)$ decreased, while the NEFA concentrations $(p<0.0001)$, cholesterol $(p<0.001)$, GGT $(p<0.001)$, and urea $(p<0.001)$ increased. There were no clinical signs indicating the development of clinical disease during the induction period. In conclusion, the protocol evaluated in this study was effective in inducing subclinical ketosis for four days, and metabolic parameters may be useful for predicting the clinical form of the disease.

Keywords: Pantaneiro genetic group. Feed restriction. Toxaemia of pregnancy. 


\section{PROTOCOLO DE INDUCCIÓN DE CETOSIS SUBCLÍNICA Y SU EFECTO SOBRE PARÁMETROS BIOQUÍMICOS EN OVEJAS GESTANTES}

\section{RESUMEN}

$\mathrm{E}$ I objetivo de este estudio fue evaluar la eficiencia de un protocolo de inducción de cetosis subclínica en ovejas del grupo genético pantaneiro en el último tercio de la gestación y su efecto sobre los parámetros metabólicos. Fueron utilizadas 13 ovejas gestantes, con 2-4 años de edad, aproximadamente 97 días de gestación, peso corporal de $40 \pm 5 \mathrm{~kg}$ y escore de condición corporal entre 2 y 3 . De los 132 a los 136 días de gestación los animales fueron sometidos a restricción alimentar del $70 \%$ de los requerimientos nutricionales de la categoría. Las muestras de sangre fueron tomadas a partir de los 97 días de gestación hasta 56 días después del parto. Fueron analizados los niveles séricos de glucosa, colesterol total, aspartato aminotransferasa (AST), gamma glutamil transferasa (GGT), albúmina, urea, fósforo, ácidos grasos no esterificados (AGNE) y beta-hidroxibutirato (BHB). Durante el período de inducción se observaron cambios metabólicos significativos en la mayoría de los metabolitos analizados. Los niveles séricos de glucosa $(p<0,0001)$ y fósforo $(p<0,001)$ disminuyeron, mientras que las concentraciones de NEFA $(p<0,0001)$, colesterol $(p<0,001)$, GGT $(p<0,001)$ y urea $(p<0,001)$ aumentaron. No fueron observados signos clínicos que indiquen el desarrollo del cuadro clínico de la enfermedad. Concluimos que el protocolo utilizado en este estudio fue eficaz para inducir la cetosis subclínica durante cuatro días y que los parámetros analizados pueden ser útiles para predecir la forma clínica de la enfermedad.

Palabras clave: Grupo genético Pantaneiro. Restricción alimentar. Toxemia da preñez.

\section{REFERÊNCIAS}

BALLOU, M. A.; GOMES, R. C.; JUCHEM, S. O.; et al. Effects of dietary supplemental fish oil during the peripartum period on blood metabolites and hepatic fatty acid compositions and total triacylglycerol concentrations of multiparous. Journal of Dairy Science, v. 92, n. 02, p. 657-669, 2009.

BOBE, G.; YOUNG, J. W.; BEITZ, D. C. Pathology, etiology, prevention, and treatment of fatty live in dairy cows. Journal of Dairy Science, v. 87, n. 10, p. 3105-3124, 2004.

BROZOS, C.; MAVROGIANNI, V. S.; FTHENAKIS, G. C. Treatment and Control of Peri-parturient Metabolic Diseases: Pregnancy Toxemis, Hypocalcemia, Hypomagnesemia. Veterinary

Clinics of North America: Food Animal Practice, v. 27, n. 1, p. 105-113, 2011. 
CALDEIRA, R. M. Monitoring the adequacy of feeding plan and nutritional status in ewes Review. Revista Portuguesa de Ciências Veterinárias, v. 100, p. 125-139, 2005.

CALDEIRA, R. M.; BELO, A. T.; SANTOS, C. C.; et al. The effect of long-term feed restriction and over-nutrition on body condition score, blood metabolites and hormonal profiles in ewes. Small Ruminant Research, v. 68, n. 03, p. 242-255, 2007.

CAMPOS, A. G. S.; AFONSO, J. A. B.; DANTAS, A. C.; et al. Estudo clínico-laboratorial da toxemia da prenhez em ovelhas: análise retrospectiva. Ciência Animal Brasileira, v. 11, n. 03, p. 623-628, 2010.

CORRÊA, M. N.; GONZÁLEZ, F. H. D.; SILVA, S. C. Transtornos Metabólicos nos Animais Domésticos. Pelotas: Editora e Gráfica Universitária/UFPEL, 2010.

EL-SHERIF, M. M. A. E.; ASSAD, F. Changes in some blood constituents of Barki ewes during pregnancy of lactation under semi-arid conditions. Small Ruminant Research, v. 40, n. 03, p. 269-277, 2001.

EMMANUEL, G. A.; NAHAPETIAN, A. Effects of 1,3-butanediol and 1,2-propanediol on blood ketone bodies and glucose in sheep fed restricted diets. Journal of Animal Science, v. 41, n. 05, p. 1468-1473, 1975.

FERREIRA, M. B.; FERNANDES, L. H.; CARMONA, R. Ovelha Pantaneira: Uma nova raça de animais com 300 anos de história. Revista Cabra e Ovelha, v. 72, p. 26-28, 2012.

GONZÁLEZ, F. H. D.; SILVA, S. C.; CERÓN, J. J.; et al. Introdução à bioquímica clínica veterinária. 2. ed. Porto Alegre: Editora da UFRGS, 2006. 364p.

GONZÁLEZ, F. H. D.; HERNÁNDEZ, F.; MADRID, J.; et al. Acid-base and electrolyte status during early induced pregnancy toxaemia in goats. The Veterinary Journal, v. 193, n. 02, p. 598-599, 2012.

GRÜNBERG, W.; STAUFENBIEL, R.; CONSTABLE, P. D.; et al. Liver phosphorus content in Holstein-Friesian cows during the transition period. Journal of Dairy Science, v. 92,n. 05, p. 2106-2117, 2009.

HARMEYER, J.; SCHLUMBOHM, C. Twin-pregnancy increases susceptibility of ewes to hypoglycaemic stress and pregnancy toxaemia. Research in Veterinary Science, v. 84, n. 02, p. 286-299, 2008.

HENZE, P. K.; BICKHARDT, H.; FUHRMANN.; et al. Spontaneous pregnancy toxaemia (ketosis) in sheep and the role of insulin. Zentralbl Veterinarmed A, v. 45, n. 05, p. 255-266, 1998. 
HOFFMAN, P. C.; ESSER, N. M.; BAUMAN, L. M.; et al. Effect of Dietary Protein on Growth and Nitrogen Balance of Holstein Heifers. Journal of Dairy Science, v. 84, n. 04, p. 843-847, 2001.

KANEKO, J.; HARVEY, W.; BRUS, M. Clinical biochemistry of domestic animals. 6. ed. San Diego: Academic Press, 2008.

LACETERA, N.; BERNABUCCI, U.; RONCHI, B.; et al. Effects of subclinical pregnancy toxemia on immune responses in sheep. American Journal of Veterinary Research, v. 62, n. 07, p. 1020-1024, 2001.

LEKATZ, L. A.; WU, G.; CATON, J. S.; et al. Maternal selenium supplementation and timing of nutrient restriction in pregnant sheep: Impacts on nutrient availability to the fetus. Journal of Animal Science, v. 89, n. 01, p. 59-76, 2011.

LEYDSON, F. F. Semiologia veterinária: a arte do diagnóstico (cães, gatos, equinos, ruminantes e silvestres). São Paulo: Roca, 2008.

MEIRA JR, E. B. S.; RIZZO, H.; BENESI, F. J.; et al. Influência dos fatores sexuais e estários sobre a proteína total, fração albumina e atividade sérica de aspartato-aminotransferase e gama-glutamiltransferase de ovinos da raça Santa Inês. Brazilian Journal of Veterinary Research and Animal Science, v. 46, n. 6, p. 448-454, 2009.

MOALLEM, U.; ROZOV, A.; GOOTWINE, E.; et al. Plasma concentrations of key metabolites and insulin in late-pregnant ewes carrying 1 to 5 fetuses. Journal of Animal Science, v. 90, n. 01, p. 318-324, 2012.

NRC - National Research Council, Nutrient requirements of dairy cattle. 7. ed. Washington: National Academic Press, 2001. 381p.

RANAWEERA, A.; FORD, E. J. H.; SAMAD, A. R. The effect of triamcinolone acetonide on plasma glucose and ketone concentration and on the total entry rate of glucose in twin pregnant hypoglycaemic ketotic sheep. Research in Veterinary Science, v. 26, n. 01, p. $12-$ 16, 1979.

REYNOLDS, C. K. Metabolism of nitrogenous compounds by ruminant liver. Journal of Nutrition, v. 122, n. 02, p. 850-854, 1992.

RUSSEL, A. J. F.; DONEY, J. M.; GUNN, R. G. Subjective assessment of body fat in live sheep. Journal of Agricultural Science, v. 72, n. 03, p. 451-454, 1969.

SAS-STATISTICAL ANALYSIS SYSTEM. Principles and Procedure of Statistics, 2. ed. McGrawHill Inc. Carry NC. 1986. 
TAGHIPOUR, B.; SEIFI, A. H.; MOHRI, M.; et al. Variations of energy related biochemical metabolites during periparturition period in fat-tailed baloochi breed sheep. Iranian Journal of Veterinary Science and Technology, v. 2, n. 2, p. 1-12, 2010.

VITTI, D. M. S. S; KEBREAB, E. Phosphorus and calcium utilization and requirements in farm animals. Oxfordshire: Cabi, 2010. 189p.

WEST, H. J. Maternal undernutrition during late pregnancy in sheep. Its relationship to maternal condition, gestation length, hepatic physiology and glucose metabolism. British Journal of Nutrition, v. 75, n. 04, p. 593-605, 1996.

Autor para correspondência: Josiane de Oliveira Feijó. Universidade Federal de Pelotas, Faculdade de Veterinária - Campus Universitário. CEP - 96010-900 - caixa postal 354, Capão do Leão (RS), Brasil. josianeofeijo@gmail.com 